\title{
INTERVENCIÓN TERAPÉUTICA CON CABALLOS VISIÓN DESDE LA PSICOLOGÍA
}

\section{Therapeutic intervention with horses vision from the psychology}

\author{
Fernanda Morey Yrigoyen*
}

\begin{abstract}
Resumen
La intervención terapéutica asistida con caballos es una herramienta integral, así como las diferentes formas de aplicación de la Psicología. Está destinada a múltiples patologías en las que se permite el uso del equino como co-terapeuta

Se realiza una pequeña descripción de las clases de terapia, funciones y condiciones, así como formas de trabajo que el Equinoterapeuta y su equipo debe contar para poder desempeñarse a cabalidad.

Se describen, las condiciones del elemento más importante de la terapia que es el caballo, sus características y condiciones de uso, en una especial y metódica observación personal en la última década de actividad profesional.
\end{abstract}

Palabras clave: Intervención terapéutica, equinoterapia, equinoterapeuta.

\begin{abstract}
Assisted therapeutic intervention with horses is a comprehensive tool, as well as different forms of application of psychology. Is intended for multiple diseases in which the use is permitted as co equine therapist

A brief description of the therapy classes, functions and conditions and ways of working that Equinoterapeuta and his team must have to fully perform is done.

It describes the conditions of the most important element of therapy is the horse, its characteristics and conditions of use, in particular, personal observation and methodical in the last decade of professional activity.
\end{abstract}

Keywords: Therapeutic intervention, equine, equinoterapeuta.

\footnotetext{
* Equinoterapeuta. Psicopedagoga. fernandamorey@gmail.com
} 


\section{INTRODUCCIÓN}

El vínculo de los caballos con la psicología, nos ha permitido encontrar mil razones de la alegría para vivir, que mostraron los caballos y que nos han ayudado a tener compromiso con metas, alegría en nuestras decisiones y obviamente, disfrutar de la vida con la naturaleza que nos rodea.

El haber sido primero jinete y luego psicóloga, me ayudó a comprender lo que un ser tan maravilloso de la naturaleza, como es el caballo, puede ayudar en muchos conceptos de vida, problemas y momentos difíciles y que muchas veces las respuestas no se encuentran en libros, pero si en el amor a lo que Dios nos da: La naturaleza con todos sus alcances y bondades, el caballo y su entorno.

La Intervención Terapéutica asistida con caballos, es para muchos un criterio aún muy nuevoen el desarrollo de apoyo al ser humano, pero si nos remontamos un poco en la historia del hombre, tendremos que simplemente observar, que el caballo y la persona siempre han estado más que unidos, y realmente con un vínculo muy arraigado y dependiente, para muchos de los logros obtenidos a través de los tiempos. Asimismo, es una respuesta complementaria, a los tradicionales modelos de intervención, tratamientos y criterios de apoyo terapéutico que existe en la actualidad.

\section{¿QUÉ ES LA EQUINOTERAPIA?}

Como menciona Gross, E. (2006) LaEquinoterapia es una terapia que utiliza el caballo como instrumento terapéutico.

En el Congreso Internacional de Monta Terapéutica, efectuado en 1988 en Toronto, Canadá, se decidió la división (aceptada posteriormente a nivel internacional) de la Equinoterapia en tres áreas:
a) Hipoterapia
b) Monta terapéutica y volting
c) Equitación como deporte para discapacitados

Cada área está dirigida a diferentes tipos de discapacidad utilizando estrategias terapéuticas distintas.

\section{NOCIONES DEL PRINCIPIO TERAPÉUTICO}

Este modelo que según Vives, J. (2004) "Es una Metodología de rehabilitación complementaria, diseñada, ejecutada y evaluada por un técnico especialista en la que utiliza el caballo y todo su entorno para intervenir sobre diferentes áreas que conforman el desarrollo integral de la persona" (pág. 23).

Este modelo, se utiliza desde hace varios siglos en la ayuda para el hombre, en sus diversas modalidades, como estímulo emocional y de corrector de conductas, posturas, en lo motor y estimulación de todas las áreas del cuerpo humano, así como en patologías mayores, donde el estímulo es tan grande que se logran respuestas insospechadas por la ciencia o la medicina.

Es una herramienta terapéutica, que se basa en el uso del caballo y sus bondades, para darle al ser humano diversas opciones de ayuda en áreas bio-psico-social, muchas veces sorprendentes, por abordarse temas de forma tan natural y espontánea, que las personas no se imaginan lo que logran experimentar con el ejercicio de esta terapia.

El caballo proporciona elementos extraordinarios, para que el terapeuta pueda aprovecharlos apropiadamente, y por ende, estimular con ello al paciente en cuestión. Cada caso es especial y único, dependiendo del criterio que se aborde, y obviamente de las características del paciente y sus redes sociales más cercanas.

Todos los elementos que brinda el caballo, deben de ser cuidadosamente estudiados por el terapeuta, para el buen uso y el cuidado que amerita el caso a tratar.

La formación del Equinoterapeuta como profesión no existe, es una especialización técnica, basada en los conocimientos profesionales de las diversas carreras que se menciona a continuación, aunque se inicia en el amor por el caballo, y en el área que se desee preparar, pudiendo ser de diversas profesiones como Psicología, Medicina, Veterinaria, Educación, Fisioterapia u otras carreras profesionales relacionadas con la salud, aunque nada se opone si se desea ayudar a través del caballo, pero dentro de los límites que el profesional destine para ese apoyo voluntario. 


\section{PRINCIPIOS TERAPÉUTICOS DEL CABALLO}

El caballo provee de tres condiciones que son de características especiales para su uso en las sesiones, como:

- El calor en el cuerpo de 38 grados en descanso, llegando a los 39 o 40 en actividad. Temperatura que permanece en el cuerpo del ser humano por seis horas posterior a la sesión, razón para su utilización en otras terapias de relajación o actividad con mejores resultados. No es necesario un pre-calentamiento cuando se trata de problemas motores.

- El movimiento tridimensional, que es similar al del ser humano en la función de la marcha, que ayuda a reconocer en casos de perdida de aquella función, y se logran recuperaciones insospechadas. Basados en la teoría de la neuroplasticidad neuronal, con la ayuda de la intervención terapéutica asistida con caballos se logran recuperar funciones que se vieron suspendidas por años, lográndose avances impresionantes, resultantes de la estimulación en base al caballo y el planeamiento de las sesiones por el profesional de la salud.

El confort y el "holding" que se experimenta con el caballo, evoca en el paciente momentos en el seno materno, promoviendo grandes beneficios, alegría y sostén a todos los seres humanos que lo recibe.

En el área emocional resulta de gran sensibilidad esta actividad, dado que en casos sobrepasa las expectativas del paciente, y hace de ello que sientan beneficios en autoestima, control de impulsos, compromiso y criterios, que se relaciona con la interacción con el caballo y el profesional que lo imparte.

- Los impulsos rítmicos del caballo son de 110 por minutos, siendo similar a los latidos del corazón del ser humano, lo que hace que la frecuencia cardiaca sea regulada en la monta terapéutica, causando beneficios de relajación y estimulación positiva en el paciente que lo recibe.

Existen personas, que no imaginaron que terminarían realizando esta actividad, y fue cuando lo experimentaron en sí mismos, que despertaron a la realidad y sensibilidad, que se logra alcanzar por medio de estos animales tan maravillosos.

\section{COMUNICACIÓN NO VERBAL Y LENGUAJE CORPORAL}

En 1967 Selvini, M psicoanalista infantil y especialista en anorexia nerviosa, fundo el Instituto de la Familia en Milán, e invitó a Luigi Boscolo, Giofranco Cecchin y Giuliana Prata; quienes fueron fuertemente influidos por Jackson, Haley, Waslawick y Bateson. En 1972, iniciaron una investigación con familias en las que algunos de sus miembros mostraban conductas psicóticas y otros contaban con un diagnóstico de esquizofrenia. Los reportes de esta investigación se encuentran en su libro Paradoja y contra paradoja.

En esta investigación las hipótesis contrales se compartían entre:

- La familia es un sistema que se gobierna a sí mismo y se autocorrige a través de las reglas que se constituyen en el tiempo por ensayo y error, indicando que es permitido o no en la familia y en la relación de cada uno de los miembros con los demás

- Toda conducta verbal o no verbal es una comunicación que afecta a otros y provoca en ellos una respuesta que a su vez es una conducta de comunicación.

En este tipo de terapias, lo primordial, esencial y condicionante de la aceptación de los casos, es la participación de la familia o cuidadores dependiendo del caso del paciente, y ello nos apoya a que el efecto terapéutico, tenga resonancia en el paciente y obviamente en los resultados de cambio en la familia.

Toda familia tiene su modus operandi, y a ello es lo que se sujeta este modelo de trabajo, al hacer participar en estas sesiones en cambios, que podrían ayudar a tener otro enfoque en las dinámicas familiares en su integridad. Depende de la colaboración de la familia en el éxito o no del trabajo que se realiza, con cada uno de los pacientes. 
El lenguaje que existe entre el hombre y el caballo es no-verbal y por medio de ello, se adecua a las órdenes que el hombre le imparte. El caballo tiene la mayoría de los movimientos a través del cuerpo similares al hombre, razón de la comunicación tan fluida que se establece entre la persona y el caballo, así como puede leer con este lenguaje no verbal, los gestos y movimientos que recibe por parte de los pacientes en general.

Los animales de terapia, son escogidos cuidadosamente de acuerdo a las condiciones que los profesionales buscamos, y usualmente son ya animales de muy buen temperamento, maduros, cuajados y dúctiles que favorece al sistema de trabajo.

Cuando se entrena un equino, se trabaja en forma ordenada y repetitiva descubriendo desde el inicio, cuales son las aptitudes de captación que cuenta el equino, para aprender las ordenes que se le da.

Las órdenes son suaves, con cariño y de menor a mayor dificultad, cosa que sucede igualmente con el hombre. Cuando el equitador es respetuoso y su entrenamiento es amable, se obtiene un equino en las mismas condiciones, cosa inversa cuando se actúa con violencia y agresividad, similar entre los seres racionales normales.

El lenguaje del cuerpo, es captado por el equino en forma natural y simbiótica, lográndose una sintonía con el entrenador, cuando este es una persona capaz y entrenada para ello.

Se dice que: No hay caballos problemáticos, sino dueños problemáticos!!

Todo vínculo, se establece de manera natural desde el nacimiento del ser, en forma no verbal entre madre e hijo, lo cual está instaurado en el cerebro desde su concepción, que con gran destreza lo disfrutamos igualmente con el caballo, gracias a la relación entre el hombre y el caballo desde su aparición en la historia del mundo.

\section{FORMAS DE PREPARACIÓN TERAPÉUTICA}

\section{Seguridad}

Es una regla de oro en esta actividad, por estar tratando con animales y con personas que tienen dificultades, se establece una razón suficiente para que se acentúe este requerimiento en el uso de esta herramienta terapéutica.

Hay parámetros internacionales de seguridad en esta forma de trabajo, y depende de cada país que se pueda o no desarrollar como se debe, pero lo más importante, es que debemos de tener todos los cuidados en lo referente a los animales y al local donde se realiza, porque necesitamos nuestros cinco sentidos para poder tratar al ser que atendemos, y de ello depende nuestro rendimiento efectivo en cada una de las sesiones.

Algo que resaltar, es que no se permiten mascotas en los centros, ya que ello distrae a los pacientes o perturba a los caballos, y más aún cuando no son del dueño del centro. Hay lugares que cohabitan perfectamente caballos, perros y gatos, porque han sido entrenados desde pequeños en el mismo lugar, pero las visitas o familiares de los pacientes no pueden brindar sus mascotas al lugar por ninguna razón.

\section{La actividad como equitador / a}

La condición más importante, es que el terapeuta sepa montar a caballo a los tres aires: paso, trote y galope. Como el nombre lo dice: Equino- terapeuta debe conocer el caballo, su manejo y los tipos de animales con los que trabaja.

La preparación como equitador es un requisito primordial del Equinoterapeuta, ya que no se podría desenvolver en las diversas formas de aplicación de la misma,al tener como herramienta principal el caballo.

El hecho de conocer los movimientos del caballo y sus características, es decir temperamento, carácter, docilidad y armonía de movimientos y respuestas a la doma, hace que el terapeuta se entrene en el conocimiento de los equinos, y obviamente, en el uso adecuado de tal o cual animal, y el correspondiente caso que se indique para ello.

El uso del equino escogido, depende del movimiento principalmente del caballo, así como el 
temperamento, que ayuda en la búsqueda de la salud integral del paciente y el diagrama de sus sesiones.

Realmente es una comunión entre la persona y el caballo, - entre el terapeuta y su co-terapeuta- que es el caballo, para poder abordar adecuadamente el uso del animal indicado para cada paciente.

\section{Conductas responsables}

Como menciona Gross, E. (2009): “QQué consideramos conductas responsables? La mayoría de estas conductas se aprenden en casa y la escuela para formarnos como miembros responsables dentro de nuestro entorno. Aprendemos a tomar responsabilidad de nosotros mismos, como asearnos, alimentarnos sanamente, organizarnos en nuestros deberes y ser ordenados. Además nos enseñan (por desgracia no siempre) a ayudar al más débil y cuidar a los seres que dependen de nosotros. La mascota en casa puede cumplir a la perfección este papel y además nos alegra por medio de su compañía.

Aunque no sea una mascota, el caballo es sumamente formativo para una persona, sea un infante o un adulto, ya que se trata de un ser vivo que depende por completo del humano, ya que es el quien debe asearlo, darle de comer y proporcionarle el espacio necesario para moverse.

Promover estas conductas responsables es el esencia el deber de cualquier instructor de equitación o Equinoterapeuta, no importa la disciplina ecuestre a practicar.

Este principio, es el que se debe instaurar en todos y cada uno de los responsables del equipo, que trabajan en el centro de Equinoterapia o de equitación, dando muy buen ejemplo con el trato hacia los equinos, orientando a los pacientes o alumnos en el correcto cuidado y limpieza de los animales y su entorno.

En la Equinoterapia el cuidado y contacto con el caballo, forma parte de la sesión, ya que ello promueve la sensibilización del paciente, así como el trabajo de los problemas sensoriales y emocionales. Fomenta la comunicación, el contacto físico y el compromiso que se busca en todos los pacientes cual sea el problema.

\section{El equipo}

Es el grupo de trabajo que se requiere, para lograr el equilibrio integral de las sesiones, y depende de la armonía de éste para avanzar en cada caso, acorde a las necesidades de los profesionales de las diferentes áreas.

El uso de la lógica plantea una formación diaria y constante durante todas y cada una de las sesiones entre los miembros del equipo. La emoción puede sorprendernos y obviamente no dejar tener objetividad en las responsabilidades de cada uno de los componentes del grupo humano de trabajo.

Como menciona Cava, R. (1999) Si se absorben las emociones negativas, deben ser liberadas de algún modo. Hemos visto que la gente se defiende de ellas de diversas formas. Pero la mayoría de estas reacciones tienen consecuencias negativas. El desafío consiste en encontrar el modo de afrontar las emociones negativas de manera constructivas.

Dos fuerzas -la lógica y la emoción- actúan en nuestro comportamiento a lo largo de nuestra vida. A menudo, empujan y tiran en direcciones opuestas. La que prevalece en un momento dado determinará cómo nos desenvolveremos con los demás y afectará a nuestra capacidad de realización. Ante cualquier circunstancia, es más fácil responder con emociones que con lógica, pero la respuesta lógica nos ayuda en condiciones difíciles a ser capaces de dominar la situación de forma constructiva.

Solo hay una persona encargada en dar las órdenes y las explicaciones tanto al paciente como a los familiares, si ello amerita. El trabajo es individual y cuenta con especificaciones que la persona que lidere el equipo, usualmente el Equinoterapeuta, el que se encarga de hacer el abordaje integral del proyecto terapéutico, las sesiones y detalles de las mismas.

En la forma de trabajo que se realiza en la aplicación terapéutica, usualmente necesitamos de tres y hasta cuatro personas, especialmente preparados en las diversas áreas, para poder abordar los temas que se plantean y programan, de acuerdo a las patologías y obviamente al carácter y personalidad del paciente. 
Dependiendo del caso y las características personales, se incluye o no a los familiares y personal de apoyo personal cuando esto amerita, obviamente si ello promueve un mejor rendimiento del proceso con el paciente.

Un equipo de trabajo se forma poco a poco, ya que se van integrando las diversas áreas de trabajo dependiendo del caso. No siempre utilizamos el mismo esquema de equipo para todos los pacientes.

En casos, donde amerita mayor énfasis en la parte motora, los fisioterapeutas son los indicados, si es que se trabaja de forma pasiva. Siempre el estudio de los casos es muy importante antes de tener el desarrollo de las sesiones, debemos analizar bien las condiciones que debemos estimular, para poder dar apoyo mayor en aquellas áreas de trabajo primordial

\section{El monitor equino}

Es una persona que conoce muy bien de caballos, que tiene manejo y experiencia de los mismos, y que además está en perfecta comunicación y compenetrado con el caso que se trata y con el terapeuta que lo aplica. Es esencial que sepa montar de forma experimentada, para realizar el entrenamiento diario de los equinos en su gimnasia y relajación imprescindible de los animales.

De él depende la armonía de la terapia, ya que un caballo bien guiado es un perfecto co-terapeuta, y por el contrario un caballo alterado o mal tratado, hace que el animal se defienda y sus defensas son "irracionales", razón de la importancia del profesional en esta área, que tiene a su cargo esta obligación y responsabilidad, para un trabajo estable y equilibrado.

\section{El equinoterapeuta}

Tiene a su cargo los pacientes desde el inicio, busca contar con el informe médico, psicológico y demás, solicitando aun radiografías o pruebas especializadas si ello lo amerita, y obviamente la aprobación del médico tratante, cuando se trata de patologías donde existe riesgo o el diagnóstico cuenta con algunos cuestionamientos.

El Equinoterapeuta debe tener formación profesional en algunas de las áreas mencionadas, predominando la Psicología, Medicina, o Fisioterapia como profesión relativa a este tipo de actividad terapéutica, ya que está vinculado con el desarrollo integral del ser en cuestión, y obviamente de la familia del paciente.

Se logran también hacer grupos de estudio de casos, con médicos Psiquiatras, Psicólogos, Neurocirujanos o Neurólogos así como también los profesores y demás terapeutas del paciente, buscando la salud integral, para que ellos también conozcan de las bondades del trabajo con caballos y poder trabajar en equipo.

El Equinoterapeuta es el que realiza la ENTREVISTA VINCULAR que es la primera entrevista, donde hace participar a la familia y sus redes más cercanas, con la finalidad de realizar un análisis integral del panorama familiar y sus interrelaciones sociales con el paciente.

Según Minuchin, C. (1989) la terapia estructural de familia, es una terapia de acción: Una transformación de la estructura familiar permitirá alguna posibilidad de cambio.

El sistema de la familia está organizado sobre la base del apoyo, regulación, alimentación y socialización de sus miembros. El terapeuta se asocia a la familia no para educarla o socializarla sino para reparar o modificar su funcionamiento para que esta pueda desarrollar las áreas con mayor eficacia.

El sistema familiar tiene propiedades de autoperpetuacion. El proceso que el terapeuta inicia se mantiene por los mecanismos de autorregulación de esta. Una vez que se ha producido un cambio, la familia lo preservará modificando la retroalimentación que continuamente califica o valida las experiencias de sus miembros.

Por medio de trabajos lúdicos y al aire libre en contacto con la naturaleza y los animales puede analizar, observar y estudiar libremente, sin intervenir, dejando que las personas logren soltar su imaginación y sus conductas, que usualmente tienen una respuesta más natural y lejos de sesgos. 
Es quien programa las sesiones de forma individual, con cada paciente. Asimismo participa todo el equipo de trabajo, y sobre todo cuando se está dando inicio a un caso diferente, y se debe tener en cuenta los matices de cada una de las sesiones especiales.

Las rutinas de algunas sesiones se anotan y se sigue con el sistema de trabajo y dependiendo de la afección, se modifica a partir de la 6ta o 10ma sesión, obviamente teniendo una evaluación previa de la respuesta del paciente.

En casos como autismo, las variaciones se deben de hacer muy sutilmente para no afectarlos con los cambios bruscos, en estos casos las alteraciones de rutinas ocasionan desconcierto y por ende rechazo o aislamiento que no se busca.

Cada paciente tiene un informe sobre las bondades que se vienen observando, así como las áreas de mayor dificultad resistentes en el avance. Usualmente, se comunica a los familiares de los avances cada tres meses, evaluando las áreas trabajadas, con la finalidad de que sean reforzados en casa o con sus pares en sus centros, cuando son pacientes institucionalizados.

A medida de informe, el trabajo que se realiza en la Asociación Nacional de Equinoterapia y Salud Integral (ANADESI), es totalmente integrador, en forma holística. Los familiares están obligados desde el inicio en participar en el desarrollo terapéutico del paciente, si no existe esta condición, no es recibido en la institución para su tratamiento. En ANADESI, no se reciben niños simplemente para impartirles las terapias sin que los familiares participen, y por lo menos estén presentes en algunas de ellas.

Salvo casos como son niños, jóvenes o adultos huérfanos, niños de la calle o casos de pacientes institucionalizados, es que ANADESI propone desde el inicio la asistencia y participación de los familiares, con el compromiso de que participen por lo menos dos o tres veces al mes, si no es que les es posible todas las sesiones.

También existen casos, en el que se les prohíbe a los familiares que participen temporalmente hasta nuevo aviso, cuando se observa que la presencia de ellos perturba el desarrollo positivo del paciente, o que hay alguna interacción por parte del familiar que distraiga demasiado al paciente. Luego el familiar es citado para poder tener una comunicación más profunda con el profesional del área que se necesita, y participar en las sesiones nuevamente.

\section{Monitores de apoyo}

Son las personas que pueden ser voluntarios o estudiantes de las carreras afines, que apoyan en el desarrollo de la terapia acompañando al terapeuta para aprender, conocer y /o practicar los estudios de Terapeuta con equinos.

Dependiendoladestrezadel voluntarioo profesional que desea apoyar, se les asigna una función en la terapia, luego de un tiempo de prueba y aprestamiento intensivo. Hay casos que tiene gran experiencia en tratamientos fisioterapéuticos y tienen la destreza de equitador, y es cuando con un entrenamiento y evaluación pasan a apoyar al Equinoterapeuta con mayor rapidez.

\section{FORMAS DE APLICACIÓN TERAPÉUTICA}

Laaplicación de esta forma de trabajo, tienealgunas particularidades, entre ellas las de Equinoterapeuta, trabajo que se realiza desde el suelo con los pacientes de forma activa o pasiva, pero siempre en contacto con el animal, que es el que provee de su movimiento y estímulo constante durante la terapia.

TERAPIA ACTIVA: es cuando el paciente colabora, comprende y puede mantenerse solo sentado encima del equino, y el terapeuta quien camina y ejercita al paciente desde el suelo, para lograr los avances que se propone en cada sesión.

Usualmente estos casos tienen un enfoque psicológico o psiquiátrico donde el criterio de baja autoestima, depresión, falta de control de impulsos, casos de psicosis, neurosis, trastornos de aprendizaje o de conducta entre otros son materia de trabajo intenso y de grandes logros personales para el paciente. También se encuentran los trastornos de conducta, aprendizaje entre otros.

TERAPIA PASIVA: es cuando el paciente no puede colaborar, y se emplea por colocar al paciente 
echado encima del caballo, de diversas posiciones dependiendo el caso a tratar. Usualmente son niños muy pequeños con problemas motores y/o retraso, que no son capaces de valerse por sí mismos sentados encima del caballo.

También se utiliza en la estimulación temprana de pequeños para el desarrollo de áreas que carecen de respuesta adecuada.

\section{HIPOTERAPIA O MONTA GEMELA}

Trabajo que se realiza desde el lomo del caballo, con el paciente sentado delante del terapeuta y que es indispensable que se ubique siempre en esa postura. Jamás el paciente irá solo, ya que se tratan problemas, usualmente motores que ameritan la presencia del terapeuta, y que el paciente obviamente, no puede mantenerse erguido solo, encima del lomo del animal.

En esta modalidad se trabajan problemas de motricidad fina y gruesa, así como derrame cerebral, parálisis, DMA, problemas musculares severos o leves, así como contracturas corporales inhabilitadoras.

\section{PSICOTERAPIA ASISTIDA CON CABALLOS- MODELO EAGALA}

Otra forma de trabajo, que se realiza con los caballos sueltos y jamás se montan. Este modelo es experiencial e incorpora al caballo como un facilitador activo para el cambio creando un paralelo con la vida de los clientes, y luego es seguido de una reflexión, análisis crítico y síntesis.

Sirve para realizar trabajos usualmente emocionales, de equipo, adicciones entre otros, donde el caballo nos sirve de herramienta espejo en las dinámicas que se realizan, consiguiéndose alternativas terapéuticas muy interesantes y de trascendencia de grupos ya sea en alumnos, familias o empresas.

Se busca que los clientes tengan iniciativas, decisiones y justifique los resultados. Los resultados son personales y constituyen la base para la experiencia y aprendizajes futuros.

Todos los modelos de trabajos con equinos, son de respuestas muy claras e inmediatas, y obviamente son trabajadas con animales especiales, confirmados en terapias que nos dan a los terapeutas, todas las garantías para el uso de los mismos en la forma adecuada y segura.

Según los Fundamentos de la Práctica del Modelo EAGALA, en su Manual para el Desentrenamiento, Quinta Edición (pág. 17)

"El Modelo EAGALA es una estructura y un estándar a llevar a cabo la Psicoterapia Asistida por Equinos (PAE). Resumiendo esta norma tenemos:

- Manejo en equipo

- Enfoque en el piso

- Orientación hacia soluciones

- Código de Ética

Las actividades del Modelo EAGALA se han diseñado para ser experienciales al incorporar al caballo como un facilitador activo en el cambio y crear un paralelo con la vida de los clientes.

La estructuración de las actividades implica enfocarse en los objetivos, necesidades y plan de tratamiento del cliente para después ser liberado, creativo y flexible con cada detalle del diseño, su escenificación y su facilitación de manera que se adapten mejor a dichas necesidades.(Nota: Los planes de tratamiento se realizan de la misma manera que en cualquier otro escenario de tratamiento) ( pag.57)

\section{CONDICIONES BÁSICAS DEL EQUIPO DE TERAPIA}

Buscando siempre la Seguridad, basada en las Condiciones básicas que el equipo debe tener como elementales en su desarrollo como profesional, mencionamos algunas:

\section{- Serenidad:}

Un profesional de la intervención terapéutica debe trasmitir ese temperamento tanto al equino como al paciente. El trabajo con los familiares, que usualmente se integran a este tipo de terapia al inicio con desconfianza y escépticos en el rendimiento de la herramienta terapéutica, debe ser muy puntual, consistente y eficiente, por el desconocimiento de las bondades de la misma, y temor que por naturaleza, 
puede despertar un contacto con un caballo, al cual no conocen.

La serenidad trasciende a través de la actitud del equipo en lo físico y lo moral, actuar con ética y responsabilidad, hace que el paciente y su entorno,confíen en lo que se está realizando con su familiar

\section{- Confianza en sí mismo:}

Debe sentirla y trasmitirla, ya que esto hace, que los participantes que lo rodeen, tengan la capacidad, de poder absorber lo mejor de la terapia y sus indicaciones.

Se debe buscar, compenetrarse con la terapia para buscar el mejor desempeño del paciente. Siempre debe pensarse en la desconfianza e inseguridad del paciente y su entorno, para procurar darles la total confianza en el desempeño de la misma

\section{- Preparación integral}

En especialidad de esta índole, el terapeuta debe ser un buen equitador, conocer los equinos, poder controlarlos y tener tacto ecuestre, siendo esto una condición imprescindible para el buen ejercicio de la misma.

Un Equinoterapeuta que no tenga manejo de equinos, no puede desempeñarse adecuadamente en esta área, y por el contrario siembra inseguridad y malestar en el entorno del paciente.

El equipo que apoya al Equinoterapeuta debe ser su personal de total confianza y muy bien entrenados en el manejo de caballos, movimientos y ayudan en caso de emergencia.

\section{- Determinación en sus funciones:}

El conocimiento del caso a tratar, así como la adecuada programación de las sesiones, hace que fluya constantemente la tranquilidad y avance del paciente y la confianza de sus familiares.

Cada integrante del equipo terapéutico, debe tener muy claras sus funciones y conocer los casos para poder colaborar en lo que se presente en las sesiones. Todo aporte es valorado por el grupo, y se acentúa cuando se va conociendo mejor al paciente en cuestión.

\section{SEGURIDAD CON EL EQUINO:}

Debe procurarse la natural transmisión de los factores anteriores para tener un co-terapeuta relajado y presto a colaborar en todo momento.

El animal, detecta la adrenalina de las personas que lo rodean, por ello se debe proveer de elementos relajantes de inicio y durante las sesiones, para poder lograr una interacción adecuada con el paciente, personal de apoyo, y los familiares.

Cuando el animal siente esa serenidad y confianza en el terapeuta y su equipo, su conducta es idónea al ambiente, surge un efecto de espejo con el equino.

El equino es un animal irracional, pero si puede ser entrenado de forma adecuada, con cariño y profesionalismo. El animal siente como es entrenado, y trasmite aquellos a los pacientes, un caballo cuidado, bien alimentado y tratado con cariño es un animal que apoya en las terapias con mucha ductilidad y paciencia.

\section{CRITERIOS DE ELECCIÓN DEL ANIMAL PARA TERAPIA}

- Tranquilo en sus movimientos.

- Temperamento tranquilo.

- Sano físicamente.

- Movimiento armonioso y marcado.

- Colaborador.

- Fuerte y rendidor .

- Dúctil, permeable al entrenamiento y las ayudas.

- Alzada o tamaño mediano.

- Volumen corporal.

- Personalidad.

- Entrenamiento previo.

No hay caballo perfecto, pero si los hay los mejores y más adecuados para la misma, así como entre los profesionales de apoyo en el equipo, quienes deben tener las condiciones idóneas para que la terapia fluya con naturalidad, alegría y relajación.

Cuando se destina al personal de apoyo a tal o cual paciente, se busca además de las condiciones mencionadas, que exista empatía entre los miembros 
del equipo, así como con los pacientes, para su mejor performance en la sesión.

\section{SEGURIDAD ENTRE LOS PROFESIONALES}

Nos debemos los profesionales, un trato en respeto, valores y ética de forma integral. Debe tenerse en cuenta el adecuado uso de mecanismos, que ayuden a los pacientes y los familiares de ellos, así como cuidar nuestra integridad física y emocional en todo momento.

Cuando se trabaja con animales debemos prever por todo nuestro entorno, y sobre todo, cuando además de ello se tiene la responsabilidad de cuidar y ayudar a una mejor calidad de vida, a cada uno de los seres que nos rodean. Comencemos por nosotros como individuos y equipo de trabajo, para poder trasmitir al resto, todo lo que tenernos internamente.

No hay mejor terapia, que cuando tanto el paciente y los familiares desean regresar y sienten que están bien considerados y cuidados en todo momento. Esto es compromiso, que debe tener toda persona, que busca apoyar al prójimo en las diversas situaciones de la vida, y más cuando hay algún problema de cualquier índole, que represente tensión y dolor en los seres humanos.

Cuando se trabaja en equipo, las órdenes y sugerencias, fluyen con naturalidad sin ocasionar perjuicios sino crecimiento y embellecimiento de las metas grupales, como debe ser en esta terapia. Si no se tiene, comunión e ideales similares en lo que se realiza, no se logra el éxito del programa final, y cada sesión se desvanece en el tiempo.

Por eso se recomienda, que el trabajo de Seguridad en los grupos humanos, se deben de tomar con todos los que participan de las terapias. Cada quien aporta lo que sabe en su responsabilidad, y así se sabe y conoce, el esfuerzo que debe poner cada uno, en su lugar de trabajo.

No hay nada mejor en esta preparación de seguridad, que las juntas constantes para conocer las carencias y las dificultades que se presentan, antes de tener a los pacientes frente al equipo. Todo debe quedar escrito, para poder solucionar cada uno de los impases, así como tener siempre, un ejercicio de herramientas preventivas, para disminuir los riesgos naturales de las interrelaciones humanas.

\section{SEGURIDAD EN EL CENTRO}

En el centro, se debe igualmente trabajar en equipo, y conocer las responsabilidades de cada uno, muy bien diagramadas, para no tener contratiempos en lo referente a responsabilidades.

El trabajo por objetivos, con cronogramas estipulados en el centro, es definitivamente una opción sumamente positiva, ya que de esa forma, se tiene a cada uno acorde a su responsabilidad, en el lugar que le corresponde, y no se yuxtaponen funciones, que es algo que denota desorden y falta de seriedad en los trabajos.

Amerita tener un plantel muy preparado y aleccionado en funciones, desde el inicio de la conformación del centro, para evitar que se establezcan conductas o vicios de conductas inadecuadas. Todo por escrito y bien detallado, ayuda a disminuir los riesgos personales, que redundan en el buen ejercicio y nombre de la institución de labores con esta especialidad.

Debe realizarse un manual de funciones donde se establezcan las normas y procedimientos del centro, para poder establecer claramente, sin darse la opción a la equivocación o a la invasión de las funciones, denotando así una buena y clara organización y administración del centro.

Todos los centros capacitados y bien organizados tienen los roles bien definidos y trabajan en equipo, que es lo que en esta actividad es indispensable.

\section{MODOS DE TRABAJO}

\section{Uso de la serotonina}

Una de las sustancias más importantes que facilitan la comunicación entre las neuronas en su punto de unión (la sinapsis) es la serotonina. A la hora de mandar un mensaje la neurona permite la salida de serotonina que actúa como un conductor bioquímico de información entre las neuronas, una vez terminado el mensaje, se recoge y guarda la serotonina. 
Diversos foros y opiniones médicas estiman que esta hormona, aparece como respuesta y causa ante problemas alimentarios, de autoestima, bienestar general, ansiedad, depresión, problemas sexuales, inconvenientes cognitivos y perceptivos.

También en estudios científicos, se han comprobado la co-relación existente entre el incorrecto funcionamiento de las comunicaciones neuronales debidas a irregularidades con la serotonina y muchas enfermedades y síndromes psicológicos como la depresión, el Trastorno obsesivo-compulsivo, etc.

La razón por la que los Inhibidores de la recaptación de serotonina funcionan en estos casos, es, como su nombre lo indica, porque una vez que han salido a enviar un mensaje no permiten que la serotonina sea recogida y guardada, de manera que la concentración de serotonina se mantiene alta entre las neuronas, permitiendo una subsecuente mejoría en las comunicaciones inter-neuronales futuras.

En el sistema nervioso central, se cree, que la serotonina representa un papel importante como neurotransmisor, en la inhibición del enfado, la inhibición de la agresión, la temperatura corporal, el humor, el sueño, el vómito, la sexualidad, y el apetito. Estas inhibiciones están relacionadas directamente con síntomas de depresión. Particularmente, los antidepresivos se ocupan de modificar los niveles de serotonina en el individuo.

Además de esto, la serotonina es también un mediador periférico de la señal. Por ejemplo, la serotonina es encontrada extensivamente en el tracto gastrointestinal (cerca del 90\%), y el principal almacén son las plaquetas en la circulación sanguínea.

La función serotoninérgica es fundamentalmente inhibitoria. Ejerce influencia sobre el sueño y se relaciona también con los estados de ánimo, las emociones y los estados depresivos.

Afecta al funcionamiento vascular así como a la frecuencia del latido cardiaco, regula la secreción de hormonas, como la del crecimiento. Cambios en el nivel de esta sustancia se asocian con desequilibrios mentales como la esquizofrenia o el autismo infantil.
También juega un papel importante en el trastorno obsesivo compulsivo, un desorden de ansiedad. Algunos hongos alucinógenos, el LSD y el MDMA actúan fuertemente en los receptores serotonínicos.

Entre las funciones fisiológicas de la serotonina destaca la inhibición de la secreción gástrica, la estimulación de la musculatura lisa y la secreción de hormonas por parte de la hipófisis. Los bajos niveles de serotonina en personas con fibromialgia explican en parte el porqué de los dolores y los problemas para dormir.

Dichos niveles bajos se han asociado también a estados agresivos, depresión y ansiedad e incluso a las migrañas, debido a que cuando los niveles de serotonina bajan, los vasos sanguíneos se dilatan. Juega un rol importante en la proliferación linfocitaria dependiendo del tipo de receptor estimulado.

Por las razones expuestas, es que en este modelo de trabajo terapéutico con caballos se hace énfasis, en el trabajo a través de la estimulación de la serotonina en el paciente, esto modifica cuantiosamente las respuestas positivas en él.

Esta forma de trabajo se utiliza en múltiples patologías que debe tener la autorización del médico tratante, y así evitar ocasionar mayores problemas en lugar de aliviarlos. En la relación compartida de los profesionales es que se logra avanzar con gran éxito de la misma, no es sino tener las precauciones y conocimiento integral del diagnóstico para poder tener la aplicación de la Equinoterapia.

\section{ESPACIOS INTEGRADOS}

Todoel trabajoes realizadoen ambientes tranquilos, agradables y obviamente bien estructurados, para poder estimular las funciones fisiológicas de la serotonina. También el uso de ejercicios lúdicos, estimula la función creativa, animándolos en sus respuestas, en sus emociones y obviamente en las vibraciones internas del ser, que es lo que la serotonina ayuda en el avance de los pacientes de diversas patologías.

Las emociones tienen un lugar especial en el desempeño de los seres humanos, y es por ello que 
se busca trabajar en ambientes relajados, positivos y siempre en "escucha", para poder dar a los pacientes el espacio que ellos merecen y obviamente a sus familiares que llegan en busca de sosiego.

Los pacientes con menos habilidades mentales tienen conductas impulsivas e inhiben la inteligencia de los demás, ya que como menciona ESPÍNDOLA, J Y ESPÍNDOLA, M (2005) "la impulsividad inhibe a la inteligencia, está ligada a los instintos y es una actitud que nos impele a hablar o actuar sin haber meditado lo suficiente para prever las consecuencias de esas acciones" (pág. 10)

En los centros de terapia, debe existir normas y procedimientos específicos en los cuales se mencionan las pautas a cumplir, por parte de los profesionales, familiares y tutores, advirtiendo a los familiares sobre el sistema de trabajo, dígase el silencio, la privacidad y respeto por cada uno de los pacientes.

Al tener la particularidad de no ser un trabajo masivo, se deberá considerar ciertos parámetros originales de trato entre los concurrentes, para que se observe la mayor tranquilidad, individualidad y paz durante las terapias para todos y cada uno de los pacientes.

Pensando siempre en el mejor desarrollo y mejorar la calidad de vida, de todos los pacientes que lleguen a ANADESI, es que se tienen estas sesiones llenas de armonía, tranquilidad y amor por ellos y nuestros caballos.

\section{REFERENCIAS}

Cava, R. (1999) Cómo tratar con personas difíciles. Ediciones Paidós Ibérica S.A.

Eagala (1999) Fundamentos del Modelo Eagala - $1^{\mathrm{a}}$ edición

Espindola J,; Espíndola M. (2005) Pensamiento Crítico -1a edición Pearson Educación de México, S.A. de C.V.

Eguiluz L. (2004) Terapia Familiar, su uso hoy en día. Editorial Pax México,

Ginger, S, Ginger A. (1993) La Gestalt, Una terapia de contacto. México D.F.: Manual Moderno.

Gross, E. (2009) Equitación y Salud. México Trillas.

Gross, E., (2006) La rehabilitación por medio del caballo. $2^{\mathrm{a}}$.ed. México: Trillas,

Kurtz, L. (2003) How to Help a Clumsy Child, Jessica Kingsley Publishers, London and Philadelphia,

Lincoln, J. (2010) Exercises for Therapeutic Riding. Ralston Store Publishing, Durango Colorado,

Vives, J. (2010) Intervención Terapéutica Asistida con caballos y Autismo. $2^{\mathrm{a}}$.ed.Revisada. Edita: Lauró projecté,

Fecha de recepción: 13 de abril del 2014

Fecha de aceptación: 16 de junio del 2014 